\title{
Synthesis and Optical Performances of a Waterborne Polyurethane-Based Polymeric Dye
}

\author{
Xianhai Hu, ${ }^{1,2}$ Xingyuan Zhang, ${ }^{2}$ and Jin Liu ${ }^{1}$ \\ ${ }^{1}$ School of Materials and Chemical Engineering, Building Energy Efficiency Research Institute, Anhui University of Architecture, \\ Hefei 230022, China \\ ${ }^{2}$ CAS Key Laboratory of Soft Matter Chemistry, Department of Polymer Science and Engineering, \\ University of Science and Technology of China, Hefei 230026, China
}

Correspondence should be addressed to Xianhai Hu; hxyh@ahjzu.edu.cn and Xingyuan Zhang; zxym@ustc.edu.cn

Received 22 October 2014; Revised 16 December 2014; Accepted 29 December 2014

Academic Editor: Jose Ramon Leiza

Copyright ( 2015 Xianhai Hu et al. This is an open access article distributed under the Creative Commons Attribution License, which permits unrestricted use, distribution, and reproduction in any medium, provided the original work is properly cited.

\begin{abstract}
A waterborne polyurethane-based polymeric dye (WPU-CFBB) was synthesized by anchoring 1, 4-bis(methylamino)anthraquinone (CFBB) to waterborne polyurethane chains. The number molecular weight, glass transition temperature, and average emulsion particle size for the polymeric dye were determined, respectively. This polymeric dye exhibited intriguing optical behaviors. The polymeric dye engendered two new absorption bands centered at about $520 \mathrm{~nm}$ and $760 \mathrm{~nm}$ if compared with CFBB in UV-vis spectra. The $760 \mathrm{~nm}$ peak showed hypsochromic shift with the decrease of average particle sizes. The polymeric dye dramatically demonstrated both hypsochromic and bathochromic effects with increasing temperature. The fluorescence intensity of the polymeric dye was much higher than that of CFBB. It was found that the fluorescence intensities would be enhanced from $20^{\circ} \mathrm{C}$ to $40^{\circ} \mathrm{C}$ and then decline from $40^{\circ} \mathrm{C}$ to $90^{\circ} \mathrm{C}$. The fluorescence of the polymeric dye emulsion was very stable and was not sensitive to quenchers.
\end{abstract}

\section{Introduction}

Polymeric dyes have attracted a great deal of attention in recent years, because of low toxicity, good colorfastness, abrasion resistance, resistance to migration, better processability, and so forth [1-5]. The polymeric dyes hold great promise in a wide range of applications. The growing interest in polymeric dyes is currently focused on anchoring monomeric dyes onto polymer matrixes to afford polymeric dyes [6-8].

Polyurethane (PU) is the most versatile polymer material to meet the highly diversified demands of modern technologies such as coatings, adhesives, fiber, foams, and thermoplastic elastomers. The development of polyurethanebased polymeric dyes has aroused some interests [9-11]. However, PU products are usually rather poor in their dyeing properties (dyeing ability and color fastness) as a result of lack of dyeing sites. A colored PU is mostly made of a mixture of pigment and polymer. However, the color is dull, with a lack of brightness and also poor abrasion resistance [12]. Moreover, elastic PU fibers are usually dyed in an energy/time consuming way, that is, at a high temperature with a long time [13].

Efforts have been exerted to improve the dyeing properties of formed membrane objects including introducing dyeable functional groups into PU, directly grafting dyestuff molecules into PU, copolymerizing with dyestuff, or fixation treatment after the dyeing process. Wang et al. synthesized a PU-based dye and found that the dyeability and thermal migration value of PU-based dye were considerably improved $[11,14]$. The formed film tended to show the glass transition point of the soft segment, lower strength, and better elongation. Chao et al. prepared a PU-based dye ionomer and found that the particle size became larger when the dye concentration was increased, and the formed film became stronger with decreased elongation [14-16]. Buruiana et al. synthesized waterborne polyurethane with various covalently bonded chromophores [17-19]. They found that tensile and thermal properties were similar to those of other elastomeric polyurethanes, but optical properties of the polymer differed from those of the monomers. 


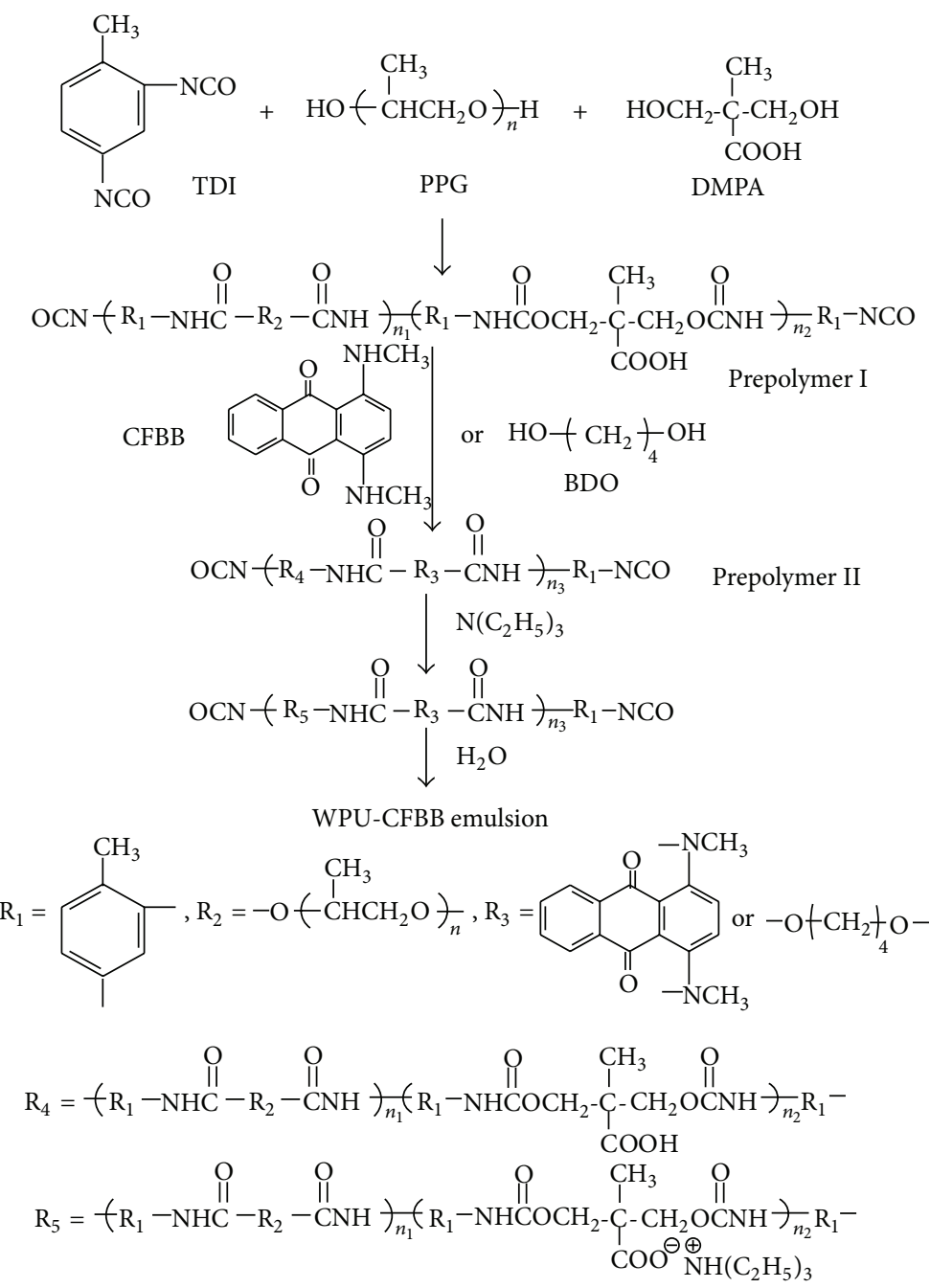

SCHEME 1: Synthesis and structure formula of WPU-CFBB or WPU.

From the above-mentioned works, study of polyurethane-based dye focused on synthesizing and modifying dyes via reaction with isocyanates to improve the dyeing properties. Other examples found in the literature usually pay attention to the performances of polymer; however, optical properties of polyurethane-based dye such as absorbance and fluorescence change with external circumstances are rarely described.

In this paper, a waterborne polyurethane-based polymeric dye (WPU-CFBB) was synthesized by anchoring 1, 4bis(methylamino)anthraquinone (CFBB, C.I.61500) to polyurethane chains. Special absorbance and fluorescence performances of WPU-CFBB were discussed in detail. WPU-CFBB can be used as an optical intelligent material with response to temperature. Because CFBB was anchored into polyurethane chains, the dye migration could be avoided, and color could be preserved permanently with brightness, solvent resistance, and good abrasion resistance as well. Additionally, WPU$\mathrm{CFBB}$ is an environment friendly material to meet the diversified demands of environmental protection. Moreover, WPUCFBB possessed the peculiar properties of optical properties, which might significantly expand the range of application in chemosensor, organic LEDs, laser active media, and so forth.

\section{Experiment}

2.1. Materials. 2, 4-Tolylene diisocyanate (TDI, Shanghai Chemical Reagent Co., Ltd., China) was distilled under reduced pressure of $10 \mathrm{mmHg}$ at $120^{\circ} \mathrm{C}$. Poly(propylene glycol) (PPG, $\mathrm{Mn}=1000 \mathrm{~g} / \mathrm{mol}$, BASF Co., Germany) was dried under the pressure of $10 \mathrm{mmHg}$ at $110^{\circ} \mathrm{C}$ for $12 \mathrm{~h}$ to remove residual water. Dimethylol propionic acid (DMPA, Aldrich Co., USA) was dried in oven reaching $120^{\circ} \mathrm{C}$ for $48 \mathrm{~h} . \mathrm{N}, \mathrm{N}$ Dimethylformamide (DMF, Aldrich Co., USA), triethylamine (TEA, Aldrich Co., USA), and acetone (Aldrich Co., USA) were distilled and kept in $4 \AA$ molecular sieve before use. Dibutyltin dilaurate (DBTDL, Aldrich Co., USA), hexane (Shanghai Chemical Reagent Co., Ltd., China), and 1, 4-bis(methylamino)anthraquinone (CFBB, BASF Co., Germany) were used as received.

2.2. Synthesis of WPU-CFBB. The synthesis of WPU-CFBB was presented in Scheme 1. TDI, PPG, DMPA, and DMF of $5 \mathrm{~mL}$ were charged into a dry four-necked flask equipped with a stirrer, thermometer, and condenser according to the molar ratio of TDI $:$ PPG $:$ DMPA $=1: 0.35: 0.28$. DBTDL was added 
to the flask under stirring (the molar ratio of TDI: DBTDL = $1: 0.02)$. The reaction was then carried out with vigorous agitation at $80^{\circ} \mathrm{C}$ under $\mathrm{N}_{2}$ atmosphere until the $\mathrm{NCO}$ content reached the theoretical value to produce NCO-terminated prepolymer I. During the reaction, the content of $-\mathrm{NCO}$ groups was monitored via a standard dibutylamine titration method [20]. In order to dilute prepolymer I, suitable amount of acetone was also added into the system to decrease the viscosity of the reaction system. Then, CFBB (the molar ratio of TDI : $\mathrm{CFBB}=1: 0.3$ ) dissolved in acetone was added and reacted with prepolymer I to form prepolymer II at $80^{\circ} \mathrm{C}$ for $6 \mathrm{~h}$. The prepolymer mixture was slowly cooled to $30^{\circ} \mathrm{C}$. TEA is just a neutralizing agent not part of the polymer backbone (the molar ratio TEA : DMPA $=1.2: 1$ ). It was added into the reaction vessel and reacted with the carboxylic group of the side chain of prepolymer II for 30 min to obtain quaternized prepolymer. Deionized water was added into the reaction mixture under vigorous agitation for $30 \mathrm{~min}$. The uniform emulsion of WPU-CFBB was obtained after acetone was removed at $50^{\circ} \mathrm{C}$ from the emulsion using a rotary evaporator under reduced pressure. The solid content of obtained WPUCFBB was about $30 \mathrm{wt} \%$. In order to compare with WPU$\mathrm{CFBB}$, waterborne polyurethane (WPU) was synthesized by means of substituting 1, 4-butanediol (BDO) for CFBB in the same conditions.

2.3. Purification of WPU-CFBB. The purification of WPUCFBB was carried out in three steps: (i) the thin film of WPUCFBB was prepared by casting the emulsion on a Teflon plate and drying at room temperature for 7 days and then in a vacuum system at $50^{\circ} \mathrm{C}$ for 2 days; (ii) the thin film of WPU-CFBB was dissolved in acetone; (iii) precipitation was carried out with hexane; the precipitated WPU-CFBB fraction was obtained after the solvent evaporation. The precipitated WPU-CFBB fraction was dissolved, precipitated, and evaporated again; the final purified film of WPU-CFBB was obtained.

2.4. Measurements. FTIR spectra (a Perkin-Elmer FTIR spectrometer) were recorded from the samples of the final purified films. ${ }^{1} \mathrm{H}$-NMR spectra were recorded on a Bruker AC 300 spectrometer operating at $300 \mathrm{MHz}$. The UV-vis spectra were recorded in a Shimadzu spectrophotometer UV2501PC. The temperature was measured with a thermocouple connected to the cell holder. Average particle size of the emulsion was determined by using a Shimadzu SALD-7101 laser particle size analyzer. Differential scanning calorimetric studies were conducted with Perkin-Elmer Pyris-1 (DSC) at a heating rate of $10^{\circ} \mathrm{C} / \mathrm{min}$ under nitrogen atmosphere. Chromatography (GPC) using a series of two linear Styragel columns HT3, HT4 and a column temperature of $35^{\circ} \mathrm{C}$ was performed on a Waters 1515 pump and Waters 2414 differential refractive index detector (set at $30^{\circ} \mathrm{C}$ ). The eluent was DMF at a flow rate of $1.0 \mathrm{~mL} / \mathrm{min}$. Fluorescence spectrometer was recorded on a Shimadzu RF-5301PC luminescence spectrometer. The temperature was measured with a thermocouple connected to the cell holder. The slit widths of monochromators were both $5 \mathrm{~nm}$.

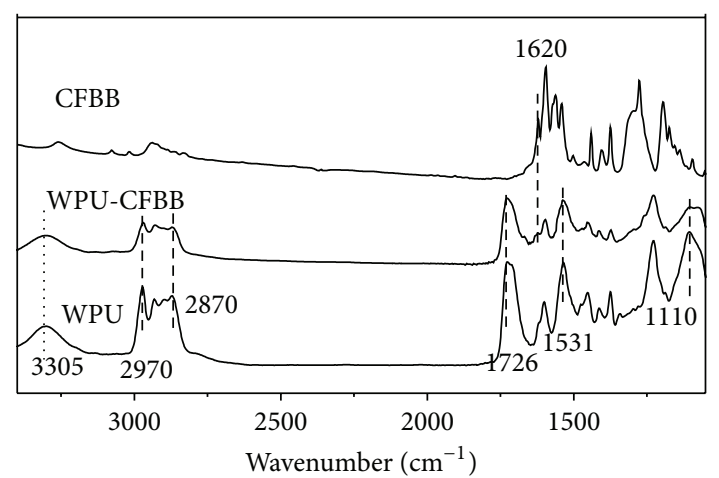

FIGURE 1: FTIR spectra of WPU, WPU-CFBB, and CFBB.

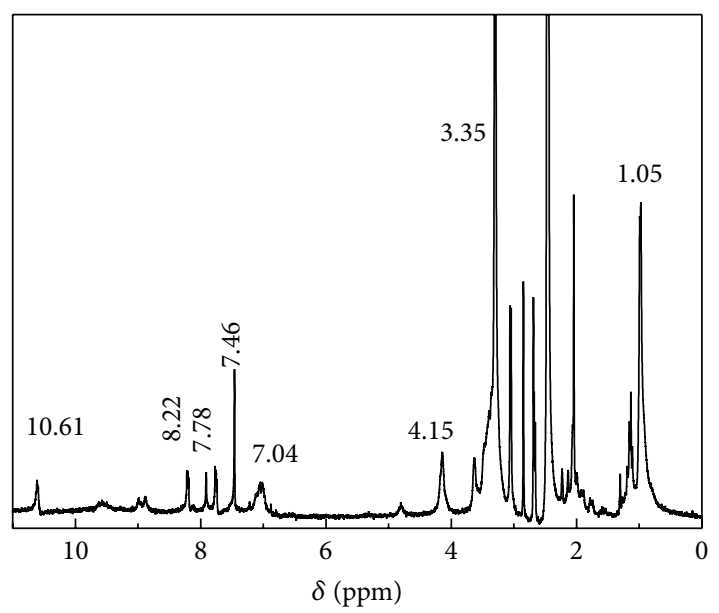

FIgURE 2: The ${ }^{1} \mathrm{H}-\mathrm{NMR}$ (DMSO- $\mathrm{d}_{6}$ ) spectrum of WPU-CFBB.

\section{Results and Discussion}

3.1. Structure Analysis of WPU-CFBB by FTIR. The expected structure of WPU-CFBB was confirmed by FTIR spectroscopy. FTIR spectra of WPU, WPU-CFBB, and CFBB were shown in Figure 1. The FTIR spectra of WPU-CFBB and WPU showed the typical peaks of polyurethane at $3305 \mathrm{~cm}^{-1}$ $[(\mathrm{NH})], 2870-2970 \mathrm{~cm}^{-1}\left[\left(\mathrm{CH}_{2}\right)\right.$ and $\left.\left(\mathrm{CH}_{3}\right)\right], 1726 \mathrm{~cm}^{-1}$ $[(\mathrm{C}=\mathrm{O})], 1531 \mathrm{~cm}^{-1}[(\mathrm{~N}-\mathrm{H})]$, and $1110 \mathrm{~cm}^{-1}[(\mathrm{C}-\mathrm{O}-\mathrm{C})]$. The characteristic peak corresponding to $\mathrm{CFBB}$ could be observed at $1620 \mathrm{~cm}^{-1}$ [(-C=O) of anthraquinone in $\left.\mathrm{CFBB}\right]$. The characteristic peak could also be observed in the spectrum of WPU-CFBB and could not be observed in WPU, indicating that CFBB has been successfully embedded into the polyurethane chains. The typical NCO peak at about $2270 \mathrm{~cm}^{-1}$ disappeared, showing that the NCO group had been completely reacted during the reaction between $\mathrm{CFBB}$ and prepolymer II.

The ${ }^{1} \mathrm{H}$-NMR (DMSO- $\mathrm{d}_{6}, \delta \mathrm{ppm}$ ) spectrum of WPUCFBB was showed in Figure 2 as follows: $10.61(\mathrm{H}, \mathrm{s}$, NHCOO); 7.04-7.46 (H, m, TDI); 4.15 (H, m, $\left.\mathrm{CH}_{2} \mathrm{OCONH}\right)$; $3.35\left(\mathrm{H}, \mathrm{m}, \mathrm{O}-\mathrm{CH}_{2}\right.$ from $\left.\mathrm{PPG}\right) ; 1.05\left(\mathrm{H}, \mathrm{m}, \mathrm{CH}\left(\mathrm{CH}_{3}\right)-\right.$ $\mathrm{CH}_{2}$ from $\left.\mathrm{PPG}\right)$; 7.78-8.22 ( $\mathrm{H}, \mathrm{m}$, anthraquinone units). Both FTIR and ${ }^{1} \mathrm{H}-\mathrm{NMR}$ demonstrated that CFBB had been attached to the polyurethane chains. 


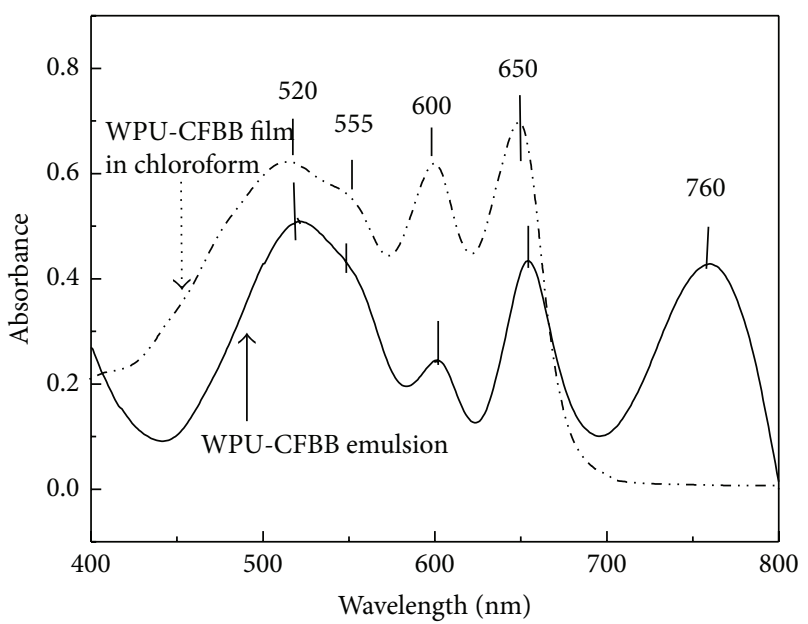

Figure 3: The UV-vis spectra of WPU-CFBB emulsion and WPUCFBB film dissolved in chloroform.

Based on comparison of the extinction coefficient of WPU-CFBB with that of CFBB, the weight percentage of $6.8 \%$ for CFBB segments in WPU-CFBB was determined by UV-vis absorption of WPU-CFBB. The number-averaged molecular weight $\left(M_{n}\right)$ of WPU-CFBB measured by GPC was about $3.3 \times 10^{4}$ and the molecular weight distribution index $\left(M_{w} / M_{n}\right)$ was 1.66. Glass transition temperature $\left(T_{g}\right)$ determined by DSC was $55^{\circ} \mathrm{C}$.

3.2. UV-Vis Spectra of WPU-CFBB. WPU-CFBB formed stable emulsion in water. WPU-CFBB emulsion showed a group of absorption bands peaked at 520,555, 600,650, and $760 \mathrm{~nm}$. WPU itself did not show any significant absorption in this range. The UV-vis spectra of WPU-CFBB emulsion and its film dissolved in chloroform were shown in Figure 3. It was found that WPU-CFBB emulsion had a new absorption band centered at $760 \mathrm{~nm}$ if compared with its film dissolved in chloroform. Intramolecular dipoles, ionization, and hydrogen bonds in the polyurethane chains were strengthened due to WPU-CFBB emulsion with electrical double surface layers through the ionization of acidic groups on the polyurethane chains, resulting in engendering new absorption in long wavelength areas corresponding to the $\pi-\pi^{*}$ transition of anthraquinone in CFBB [21].

The UV-vis spectra of CFBB in chloroform and WPUCFBB film dissolved in chloroform were shown in Figure 4. WPU-CFBB showed three typical absorption bands centered at 555,600 , and $650 \mathrm{~nm}$ associated with the anthraquinone unit of CFBB, which further confirmed that CFBB was attached to the polyurethane chains. WPU-CFBB film dissolved in chloroform showed a new absorption band centered at $520 \mathrm{~nm}$ compared with CFBB, corresponding to the $\pi-\pi^{*}$ transition resulting from decreasing $\pi$ energy grade and enhancing $\pi^{*}$ energy grade of anthraquinone unit through inductive effect [21].

In order to demonstrate the relations of two new absorption maxima to the sizes of emulsions, a series of WPUCFBB emulsions with different particle sizes were prepared by

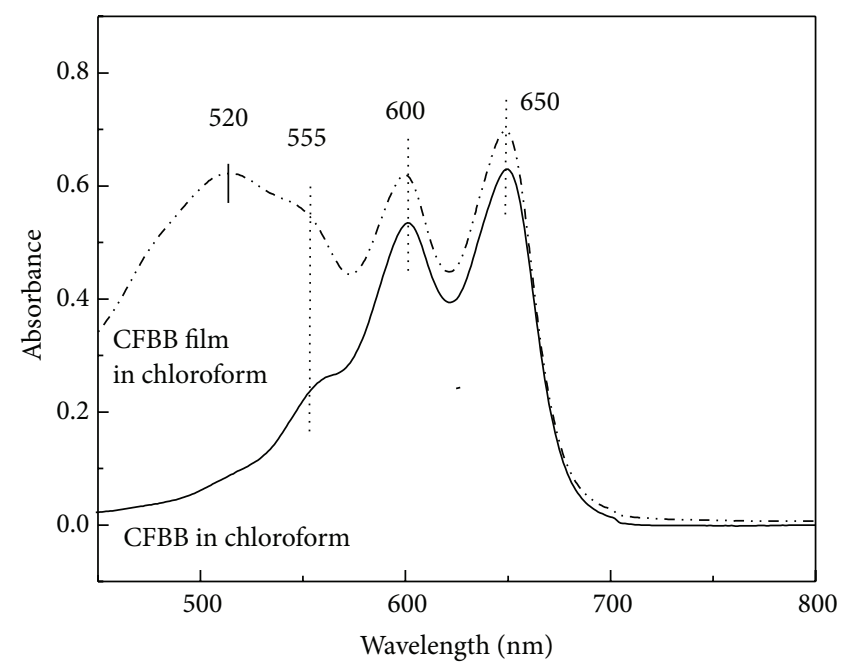

FIGURE 4: The UV-vis spectra of CFBB in chloroform and WPUCFBB film dissolved in chloroform.

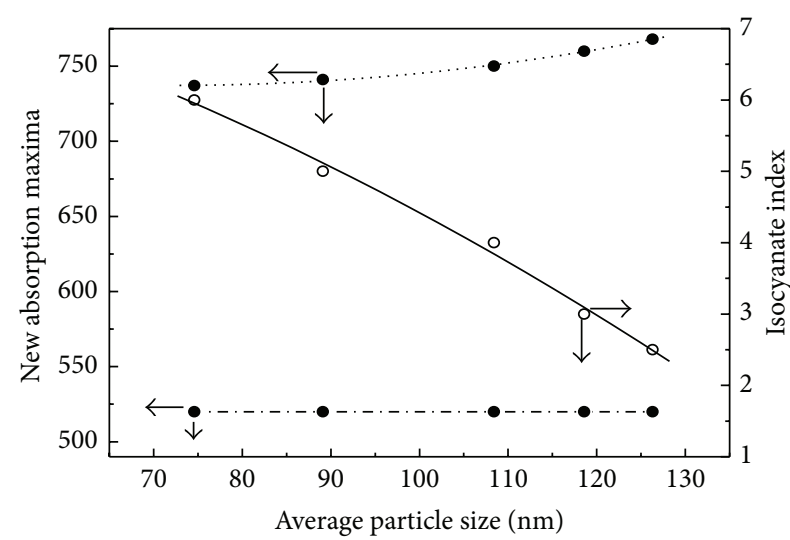

FIGURE 5: Relationships between the isocyanate index, the average particle size, and the two new absorption maxima for WPU-CFBB emulsion.

changing isocyanate index according to $6: 1,5: 1,4: 1$, and $3: 1$ molar ratio of TDI to PPG during the synthesis. Generally, PPG was added to react with TDI to form prepolymer. The isocyanate index could determine directly average particle size of the emulsions $[22,23]$. The correlations between the isocyanate index, the average particle sizes, and the two new absorption maxima for WPU-CFBB emulsion were Figure 5. With the decrease of the isocyanate index, the average particle sizes of emulsion were significantly enlarged from $75 \mathrm{~nm}$ to $126 \mathrm{~nm}$. The new absorption maxima of $520 \mathrm{~nm}$ did not change with enlarged average particle sizes. However, the new absorption maxima of $760 \mathrm{~nm}$ were obviously hypsochromic shift with decreased average particle sizes from $760 \mathrm{~nm}$ to $740 \mathrm{~nm}$. The phenomenon could be attributed to weakened microzones of CFBB segments in the decreased average particle sizes of WPU-CFBB emulsions with the isocyanate index increased.

Normally, absorption spectrum displayed bathochromic effect along with increasing temperature. However, WPUCFBB latex dramatically demonstrated both hypsochromic 


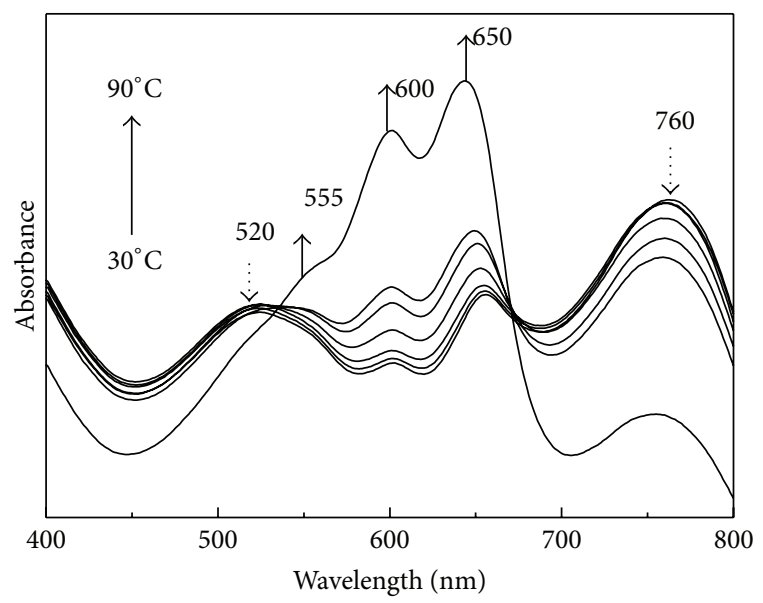

Figure 6: The UV-vis spectra of WPU-CFBB emulsion varying with temperature.

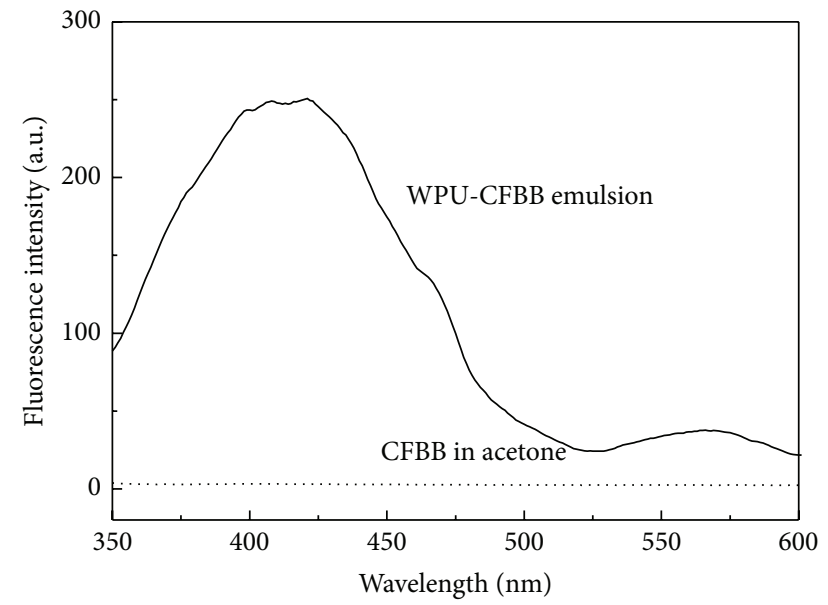

FIGURE 7: Fluorescence spectra of WPU-CFBB and CFBB in acetone under $325 \mathrm{~nm}$ excitation.

and bathochromic effects with increasing temperature. As could be seen from Figure 6, three strong absorption bands centered at 555, 600, and $650 \mathrm{~nm}$ associated with the anthraquinone unit showed bathochromic effects along with temperature increased from 30 to $90^{\circ} \mathrm{C}$, corresponding to the $\pi-\pi^{*}$ transition, whereas bathochromic effects are much stronger from 80 to $90^{\circ} \mathrm{C}$. The two new absorbance bands of WPU-CFBB latex centered at 520 and $760 \mathrm{~nm}$ unusually showed hypsochromic effect, which were attributed to reducing aggregation tendency of WPU-CFBB and decreasing the hydrogen bonding in WPU-CFBB with increasing temperature $[24,25]$.

3.3. Fluorescence of $W P U-C F B B$. The fluorescence spectra of WPU-CFBB emulsion and CFBB in acetone were shown in Figure 7. The fluorescence intensity of WPU-CFBB was much higher than that of $\mathrm{CFBB}$ at the same molar concentrations of chromophores. In fact, CFBB displayed very weak fluorescence emission. The fluorescence quantum yield $\left(\Phi_{F}\right)$ of WPU-CFBB and CFBB was determined using Rhodamine B

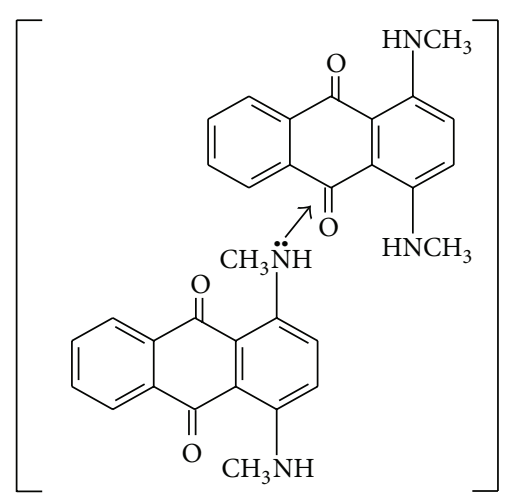

Scheme 2: Intermolecular excimer of CFBB.

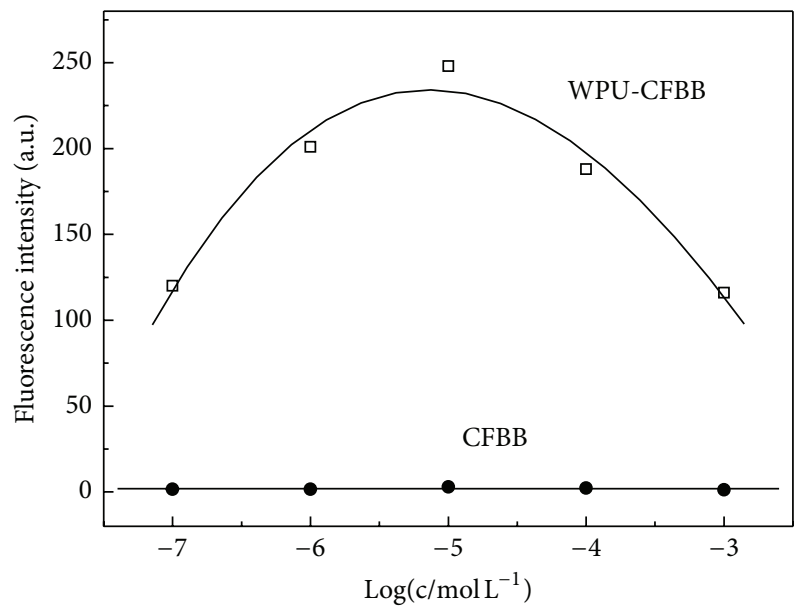

FIGURE 8: Fluorescence intensity of WPU-CFBB and CFBB at different concentrations under $325 \mathrm{~nm}$ excitation.

$\left(\Phi_{0}=0.89\right)$ as the reference. The calculated result showed that $\Phi_{F}$ of WPU-CFBB was 0.18 while that of CFBB in acetone is 0.01 . The $\Phi_{F}$ of WPU-CFBB emulsion was 18 times higher than that of CFBB in acetone.

The weak fluorescent emission of CFBB was not due to the so-called "concentrational self-quenching effect" but because of "structural self-quenching effect" $[26,27]$. This phenomenon was attributed to the intermolecular excimers formation of CFBB between electron-donating imino group and electron-accepting carbon-oxygen double bond due to the molecular structure of CFBB containing both electronwithdrawing and electron-donating groups, as illustrated in Scheme 2. The formation of abundant excimers caused fluorescence quenching and engendered a weak fluorescence emission.

In order to prove that such a low fluorescence intensity of CFBB was not due to the "concentrational self-quenching effect," the fluorescence intensity of WPU-CFBB and CFBB was measured in a wide concentration range of $10^{-7}$ to $10^{-3} \mathrm{M}$. Figure 8 showed the relationships between the fluorescence intensity and the solution concentration. The fluorescence intensity displayed a maximum at the concentration of about $10^{-5} \mathrm{M}$ for WPU-CFBB. It was clear that the fluorescence intensity of WPU-CFBB was always much higher than that 

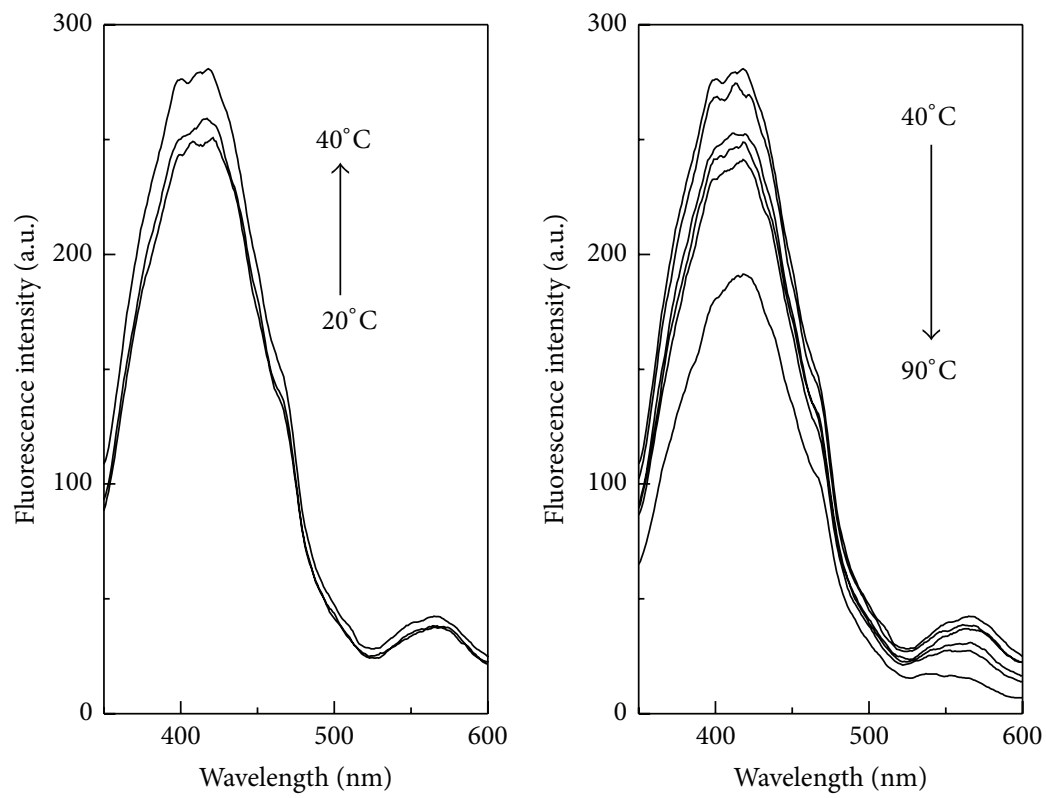

FIGURE 9: The fluorescence spectra of WPU-CFBB under different temperature under $325 \mathrm{~nm}$ excitation.

of CFBB at all concentrations. The concentration changing could not enlarge visibly the fluorescence intensity of CFBB.

The fluorescence intensity of WPU-CFBB was greatly enhanced comparing with that of $\mathrm{CFBB}$, which was mainly attributed to the following two factors. First, CFBB was anchored to the polyurethane chains, which hindered the formation of excimers among CFBB. Furthermore, the intramolecular rotation and vibration of CFBB were restricted, resulting in a decrease in nonradiative transition and thus an increase in radiative transition process $[28,29]$. Secondly, WPU-CFBB formed emulsion with electrical double surface layers due to the ionization of acidic groups, leading to augmented light absorption area [30]. In addition, the CFBB segments of WPU-CFBB were inside electrical double layer; thus the mobility of $\mathrm{CFBB}$ was decreased and $\mathrm{CFBB}$ was shielded from possible quenchers $[31,32]$.

Generally speaking, fluorescent intensity would gradually decline in solution along with increasing temperature. However, WPU-CFBB latex exhibited intriguing optical behavior. As shown in Figure 9, the fluorescence intensity of WPU$\mathrm{CFBB}$ was enhanced as the temperature increased from 20 to $40^{\circ} \mathrm{C}$ and then declined from 40 to $90^{\circ} \mathrm{C}$. The enhanced fluorescence intensity from 20 to $40^{\circ} \mathrm{C}$ could be attributed mainly to a lessened energy difference between $S_{1}$ and $T_{1}$ by thermal energy and decreased hydrogen bonding in WPUCFBB with increasing temperature [33]. WPU-CFBB can be used as an optical intelligent material with response to temperature.

The fluorescence of WPU-CFBB was very stable during storage. Hardly any difference of fluorescence intensity was observed after 30 days. Furthermore, fluorescence of WPUCFBB decreased only a little after some quenchers (e.g., hydroquinone) were added into WPU-CFBB.

The fluorescence of WPU-CFBB was regularly quenched by adding the hydroquinone (Figure 10(a)). Fluorescence

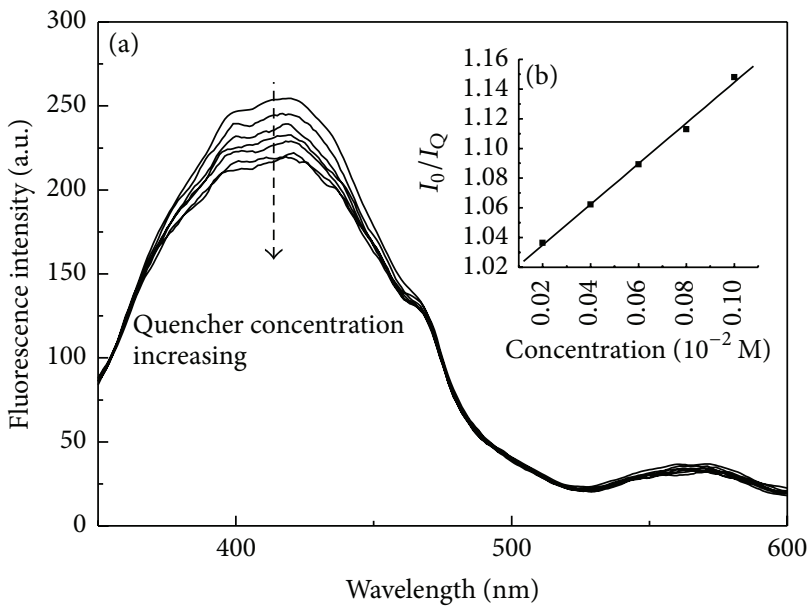

FIGURE 10: (a) Fluorescent spectra of WPU-CFBB emulsion in increasing quencher concentration of hydroquinone under $325 \mathrm{~nm}$ excitation; (b) the plot of $I_{0} / I_{\mathrm{Q}}$ versus the concentration of hydroquinone $[Q]$.

quenching efficiency of quencher hydroquinone can be analyzed according to the Stern-Volmer equation:

$$
\frac{I_{0}}{I_{\mathrm{Q}}}=1+K_{\mathrm{SV}}[\mathrm{Q}]
$$

where $I_{0}$ is fluorescence intensity of WPU-CFBB emulsion without quencher while $I_{Q}$ is the fluorescence intensity containing quencher; $[Q]$ is the concentration of quencher, and $K_{\mathrm{SV}}$ is dynamic quenching constant. $K_{\mathrm{SV}}$ value indicates the fluorescence quenching efficiency. Figure 10(b) demonstrated a linear relationship between the $I_{0} / I_{Q}$ and $[Q]$. The very small dynamic quenching constant $K_{\mathrm{SV}}$ of $14.8 \mathrm{M}^{-1}$ could be determined from the slope of the line, indicating clearly 
the fluorescence stability of WPU-CFBB emulsions. The CFBB segments of WPU-CFBB were inside electrical double layer due to the ionization of acidic groups on the polyurethane chains; thus CFBB segments were shielded from quenchers. Generally speaking, the fluorescence of WPU-CFBB was not sensitive to quenchers.

\section{Conclusions}

A waterborne polyurethane-based dye WPU-CFBB was prepared by chemically anchoring CFBB into waterborne polyurethane chains. The number average molecular weight and its distribution index and glass transition temperature for WPU-CFBB were determined to be $3.3 \times 10^{4}, 1.66$, and $55^{\circ} \mathrm{C}$, respectively. Compared with $\mathrm{UV}$-vis spectrum of $\mathrm{CFBB}$, WPU-CFBB emulsion demonstrated two new absorption bands centered at about $520 \mathrm{~nm}$ and $760 \mathrm{~nm}$, respectively. The $520 \mathrm{~nm}$ peak was no change with enlarged average particle sizes. However, the $760 \mathrm{~nm}$ peak showed hypsochromic shift with the decrease of average particle sizes. WPU-CFBB demonstrated both hypsochromic and bathochromic effects with increasing temperature. The fluorescence intensity of WPU-CFBB was prominently enhanced in comparison with $\mathrm{CFBB}$, which was mainly attributed to the hindered formation of excimers among CFBB and the augmented light absorption area. The fluorescence intensity of WPU-CFBB was enhanced from 20 to $40^{\circ} \mathrm{C}$ and then declined from 40 to $90^{\circ} \mathrm{C}$. The enhanced fluorescence intensity from 20 to $40^{\circ} \mathrm{C}$ could be mainly attributed to a lessened energy difference between $S_{1}$ and $T_{1}$ and decreased hydrogen bonding in WPUCFBB. WPU-CFBB can be used as an optical intelligent material with response to temperature. In addition, the fluorescence of WPU-CFBB emulsions was very stable and was not sensitive to quenchers according to a fluorescence quenching analysis.

\section{Conflict of Interests}

The authors declare that there is no conflict of interests regarding the publication of this paper.

\section{Acknowledgments}

Financial support from the National High Technology Research and Development Program of China (no. 2014AAQ00294), the National Natural Science Foundation of China (no. 51073144), and the Natural Science Foundation of Anhui Education Department (no. KJ2014A045) is acknowledged.

\section{References}

[1] X. Q. Zhang, X. Y. Zhang, B. Yang et al., "A novel method for preparing AIE dye based cross-linked fluorescent polymeric nanoparticles for cell imaging," Polymer Chemistry, vol. 5, no. 3, pp. 683-688, 2014.

[2] J. L. Wang and L. M. Leung, "Synthesis and characterization of highly soluble blue emitting poly(2-vinylanthracene) with 9,10di(2-naphthalenyl) and 9,10-di(3-quinolinyl) substituents," Dyes and Pigments, vol. 99, no. 1, pp. 105-115, 2013.
[3] B. T. Li, J. Shen, Y. M. Jiang, J. Wang, and C. Kan, "Preparation and properties of covalently colored polymer latex based on a new anthraquinone monomer," Journal of Applied Polymer Science, vol. 129, no. 3, pp. 1484-1490, 2013.

[4] X. L. Jin, X. G. Yu, W. Zhang, J. Zhou, G. P. Tang, and C. F. Zhong, "Synthesis and photovoltaic properties of main chain polymeric metal complexes containing 8-hydroxyquinoline metal complexes conjugating alkyl fluorene or alkoxy benzene by $\mathrm{CN}$ bridge for dye-sensitized solar cells," Polymer Composites, vol. 34, pp. 1629-1639, 2013.

[5] G. A. Lindsay, M. C. Davis, A. P. Chafin et al., "Electro-optic polymeric films of phenylethynyl dyes with transparency in the blue region of the visible spectrum," Optical Materials, vol. 32, no. 2, pp. 302-310, 2009.

[6] L. Guo, J. Deng, L. Zhang, Q. Xiu, G. Wen, and C. Zhong, "Synthesis and applications of 3,6-carbazole-based conjugated sidechain copolymers containing complexes of 1,10-phenanthroline with $\mathrm{Zn}(\mathrm{II}), \mathrm{Cd}(\mathrm{II})$ and $\mathrm{Ni}(\mathrm{II})$ for dye-sensitized solar cells," Dyes and Pigments, vol. 92, no. 3, pp. 1062-1068, 2012.

[7] M. Gómez-Tardajos, J. P. Pino-Pinto, C. Díaz-Soto et al., "Confinement of 5,10,15,20-tetrakis-(4-sulfonatophenyl)-porphyrin in novel poly(vinylpyrrolidone)s modified with aromatic amines," Dyes and Pigments, vol. 99, no. 3, pp. 759-770, 2013.

[8] X. Hu, X. Zhang, J. Liu, and J. Dai, "Synthesis, characterization and fluorescence performance of a waterborne polyurethanebased fluorescent dye 4-amino- $N$-cyclohexyl-1,8-naphthalimide, WPU-ACN," Polymer International, vol. 63, no. 3, pp. 453458, 2014.

[9] Z. A. Li, W. B. Wu, P. Hu et al., "Click modification of azocontaining polyurethanes through polymer reaction: convenient, adjustable structure and enhanced nonlinear optical properties," Dyes and Pigments, vol. 81, no. 3, pp. 264-272, 2009.

[10] X. H. Hu, X. Y. Zhang, J. Liu, and J. B. Dai, "Synthesis and fluorescence enhancement behavior of a novel fluorescent aqueous polyurethane emulsion DDAQ-TDI-PU," Chinese Chemical Letters, vol. 23, no. 7, pp. 855-858, 2012.

[11] E. C. Buruiana, V. Melinte, T. Buruiana, B. C. Simionescu, T. Lippert, and L. Urech, "Synthesis and photochemical investigations of novel bistriazene polyurethanes," Journal of Photochemistry and Photobiology A: Chemistry, vol. 186, no. 2-3, pp. 270277, 2007.

[12] A. E. Tret'yakova, V. V. Safonov, and A. Y. Yusina, "Study of processes of dyeing polyurethane fibres by various classes of dyes," Fibre Chemistry, vol. 44, no. 5, pp. 284-287, 2013.

[13] J. J. Lee, Z. K. Chi, Y. M. Kuo, and D. Y. Chao, "Effect of the concentration of dye on the physical property of dye-based polyurethane ionomer," Journal of Applied Polymer Science, vol. 57, no. 8, pp. 1005-1011, 1995.

[14] H.-H. Wang and I.-S. Tzun, "Modified polyurethane with a covalent bond of dye molecule," Journal of Applied Polymer Science, vol. 73, no. 2, pp. 245-253, 1999.

[15] Y. M. Kuo, K. Y. Ku, H. C. Lin, N. H. Wang, C. P. Chwang, and D. Y. Chao, "A study of colored siloxane-based polyurethane ionomer," Journal of Applied Polymer Science, vol. 69, no. 11, pp. 2097-2105, 1998

[16] M. S. Yen, P. Y. Chen, and H. C. Tsai, "Synthesis, properties, and dyeing application of nonionic waterborne polyurethanes with different chain length of ethyldiamines as the chain extender," Journal of Applied Polymer Science, vol. 90, no. 10, pp. 28242833, 2003. 
[17] E. C. Buruiana, V. Melinte, T. Buruiana, T. Lippert, H. Yoshikawa, and H. Mashuhara, "Synthesis and characterisation of new hard polyurethanes with triazene pendants," Journal of Photochemistry and Photobiology A: Chemistry, vol. 171, no. 3, pp. 261-267, 2005.

[18] E. C. Buruiana, T. Buruiana, G. Strat, and M. Strat, "Synthesis and fluorescence of polyurethane cationomers N-modified with a stilbene chromophore," Journal of Photochemistry and Photobiology A: Chemistry, vol. 162, no. 1, pp. 23-31, 2004.

[19] E. C. Buruiana, T. Buruiana, G. Strat, and M. Strat, "Synthesis and optical properties of new polyurethane cationomers with anchored stilbene chromophores," Journal of Polymer Science, Part A: Polymer Chemistry, vol. 40, no. 11, pp. 1918-1928, 2002.

[20] "standard test method for isocyanate groups in urethane materials or prepolymers," ASTM D2572-03, ASTM, West Conshohocken, Pa, USA, 2003.

[21] A. V. Yatsenko, V. A. Tafeenko, V. N. Zakharov, S. I. Popov, and H. Schenk, "An INDO/S study of the second $\pi-\pi^{*}$ transition in the spectra of 1,4-diaminoanthraquinones responsible for their green hue," Dyes and Pigments, vol. 37, pp. 273-282, 1998.

[22] J. Y. Jang, Y. K. Jhon, I. W. Cheong, and J. H. Kim, "Effect of process variables on molecular weight and mechanical properties of water-based polyurethane dispersion," Colloids and Surfaces A: Physicochemical and Engineering Aspects, vol. 196, no. 2-3, pp. 135-143, 2002.

[23] B. K. Kim, T. K. Kim, and H. M. Jeong, "Aqueous dispersion of polyurethane anionomers from $\mathrm{H}_{12} \mathrm{MDI} / \mathrm{IPDI}, \mathrm{PCL}, \mathrm{BD}$, and DMPA," Journal of Applied Polymer Science, vol. 53, no. 3, pp. 371-378, 1994.

[24] H. Meier, R. Petermann, and J. Gerold, "Bathochromic or hypsochromic effects via the extension of conjugation: a study of stilbenoid squaraines," Chemical Communications, vol. 11, pp. 977-978, 1999.

[25] H. Meier, J. Gerold, H. Kolshorn, and B. Mühling, "Extension of conjugation leading to bathochromic or hypsochromic effects in OPV series," Chemistry - A European Journal, vol. 10, no. 2, pp. 360-370, 2004.

[26] X. H. Hu, X. Y. Zhang, and J. B. Dai, "Synthesis and characterization of a novel waterborne stilbene-based polyurethane fluorescent brightener," Chinese Chemical Letters, vol. 22, no. 8, pp. 997-1000, 2011.

[27] F.-S. Du, Y. Zhou, Z.-C. Li, and F.-M. Li, "Vinyl monomers bearing chromophore moieties and their polymers. XII. Synthesis and fluorescence behavior of vinyloxy monomers having 1,8naphthalimide moiety with different spacer lengths and their polymers," Polymers for Advanced Technologies, vol. 11, no. 8-12, pp. 798-804, 2000.

[28] X.-H. Hu, X.-Y. Zhang, J.-B. Dai, and J. Liu, "Synthesis and fluorescent investigations of VBL-based waterborne polyurethane dye," Journal of Luminescence, vol. 131, no. 10, pp. 2160-2165, 2011.

[29] Z. Li, Y. Dong, B. Mi et al., "Structural control of the photoluminescence of silole regioisomers and their utility as sensitive regiodiscriminating chemosensors and efficient electroluminescent materials," The Journal of Physical Chemistry B, vol. 109, no. 20, pp. 10061-10066, 2005.

[30] M. Kawa and J. M. J. Fréchet, "Self-assembled lanthanide-cored dendrimer complexes: enhancement of the luminescence properties of lanthanide ions through site-isolation and antenna effects," Chemistry of Materials, vol. 10, no. 1, pp. 286-296, 1998.
[31] J. Virtanen, H. Lemmetyinen, and H. Tenhu, "Fluorescence and EPR studies on the collapse of poly( $\mathrm{N}$-isopropyl acrylamide)g-poly(ethylene oxide) in water," Polymer, vol. 42, no. 23, pp. 9487-9493, 2001.

[32] H. Xianhai, X. Zhang, J. Liu, and J. Dai, "Synthesis, characterization and fluorescence performance of a waterborne polyurethane-based polymeric dye," Journal of Luminescence, vol. 142, pp. 23-27, 2013.

[33] D. K. Kunimitsu, A. Y. Woody, E. R. Stimson, and H. A. Scheraga, "Thermodynamic data from fluorescence spectra. II. Hydrophobic bond formation in binary complexes," The Journal of Physical Chemistry, vol. 72, no. 3, pp. 856-866, 1968. 

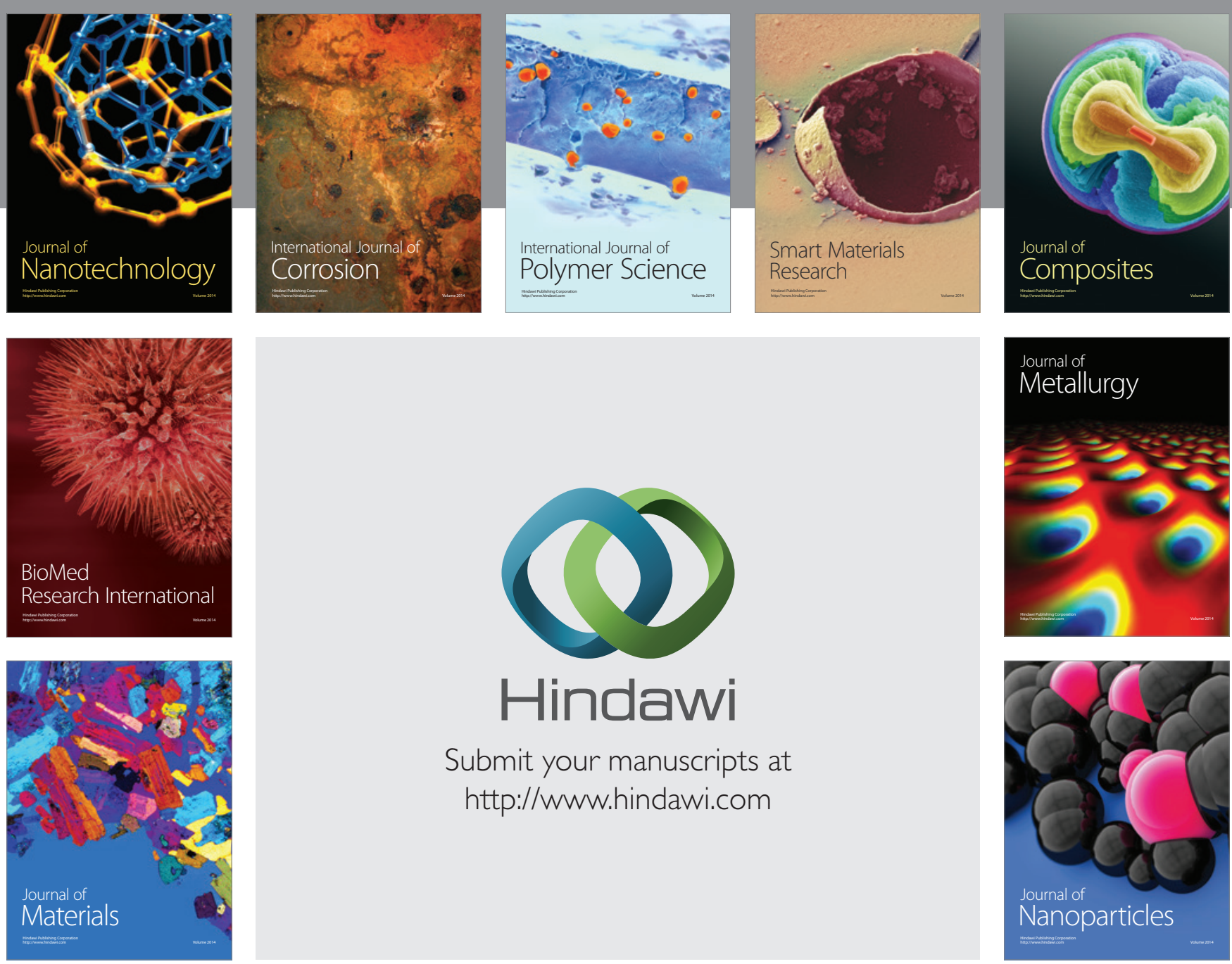

Submit your manuscripts at http://www.hindawi.com
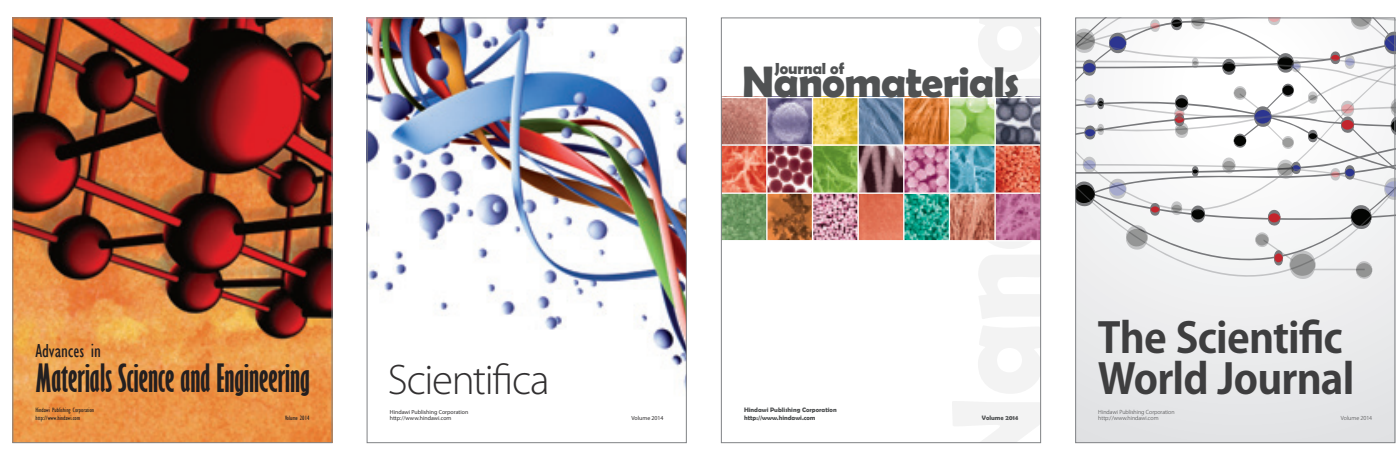

\section{The Scientific World Journal}
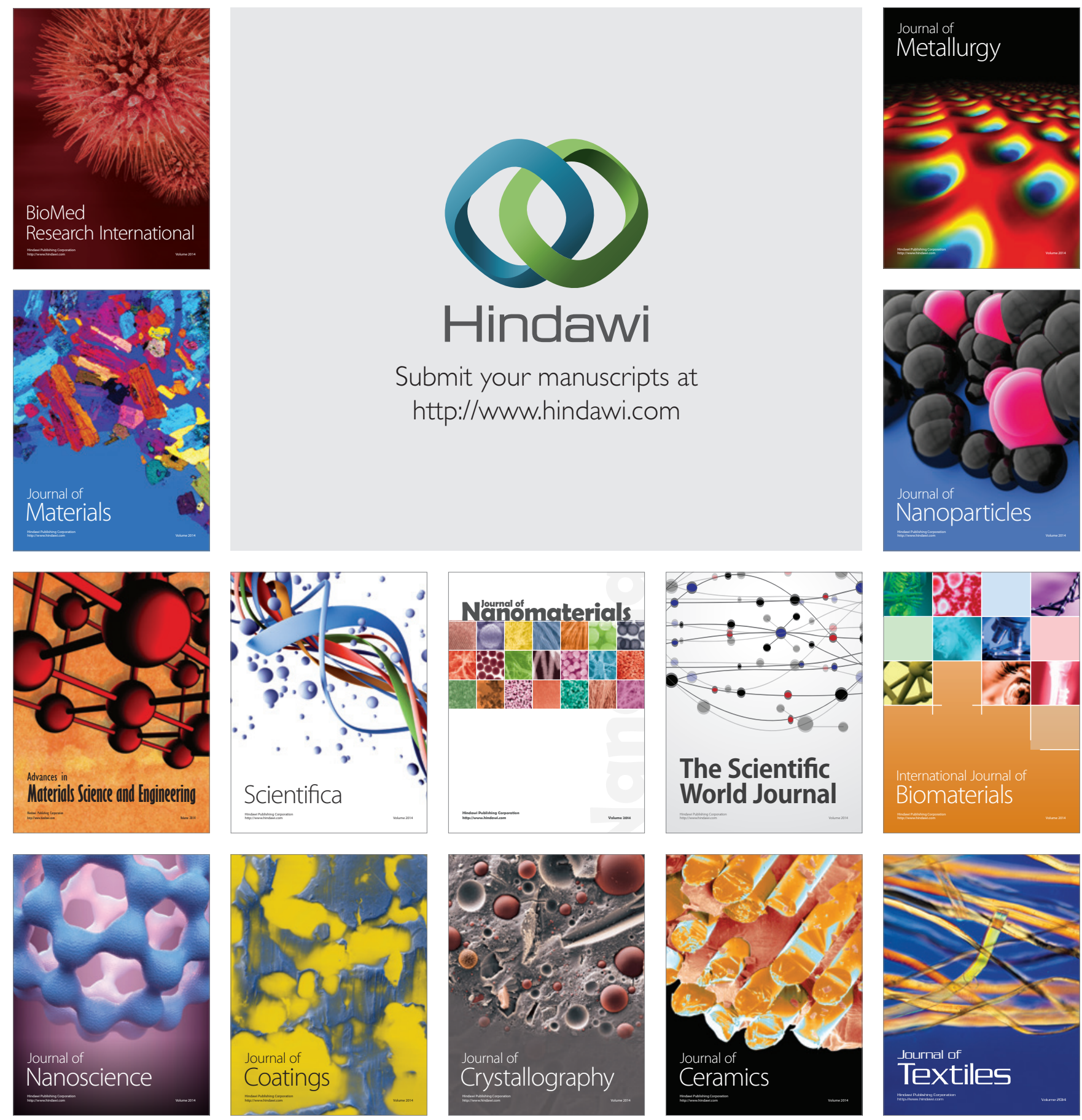